\title{
BOUNDARY BEHAVIOR OF THE BERGMAN METRIC
}

\author{
BO-YONG CHEN
}

\begin{abstract}
Let $\Omega$ be a bounded pseudoconvex domain in $\mathbf{C}^{n}$. We give sufficient conditions for the Bergman metric to go to infinity uniformly at some boundary point, which is stated by the existence of a Hölder continuous plurisubharmonic peak function at this point or the verification of property $(P)$ (in the sense of Coman) which is characterized by the pluricomplex Green function.
\end{abstract}

\section{$\S 1$. Introduction}

Let $\Omega$ be a bounded domain in $\mathbf{C}^{n}$, and let $K_{\Omega}(z)$ be the Bergman kernel function on $\Omega$. The Bergman metric is defined by

$$
B_{\Omega}(z ; X)=\left(\sum_{j . k=1}^{n} \frac{\partial^{2} \log K_{\Omega}(z)}{\partial z_{j} \partial \bar{z}_{k}} X_{j} \bar{X}_{k}\right)^{1 / 2}
$$

where $X=\sum_{j=1}^{n} X_{j} \partial / \partial z_{j} \in T^{1,0}\left(\mathbf{C}^{n}\right)$. Recently, it was proved independently by Blocki-Pflug [2] and Herbort [16] that the Bergman metric of an arbitrary bounded hyperconvex domain is complete. This is a partial but satisfactory answer to the old problem of Kobayashi [19]: Which bounded pseudoconvex domain is Bergman complete? This problem has been extensively studied (see also [3], [17], [21], [22]). However, the Bergman completeness does not guarantee that the Bergman metric tends to infinity uniformly at the boundary. A polydisc is the most simple example. Notice that such a domain does not admit a plurisubharmonic (psh for short) peak function at each boundary point. Recall that a function $\rho$ is called a psh peak function at a boundary point $w_{0}$ of a domain $\Omega$ if $\rho$ is psh in $\Omega$, continuous on $\bar{\Omega}$ with $\rho\left(w_{0}\right)=0$ and $\rho(z)<0$ for all $z \in \bar{\Omega} \backslash\left\{w_{0}\right\}$. It is natural to ask whether the Bergman metric of a bounded pseudoconvex domain go to infinity uniformly at a boundary point if there is a psh peak function at

Received June 26, 2000.

2000 Mathematics Subject Classification: 32A25. 
this point. The main purpose of this paper is to give the following partial result:

THEOREM 1. Let $\Omega$ be a bounded pseudoconvex domain in $\mathbf{C}^{n}$ and let $w_{0} \in \partial \Omega$. Suppose that there exists a psh peak function $\rho$ for $\Omega$ at $w_{0}$ which is Hölder continuous at $w_{0}$, that $i s$, there exist constants $c, \gamma>0$ such that $\rho(z) \geq-c\left|z-w_{0}\right|^{\gamma}$ holds for all $z \in \bar{\Omega}$. Then one has

$$
\inf _{0 \neq X \in T^{1,0}\left(\mathbf{C}^{n}\right)} B_{\Omega}(z ; X) /|X| \rightarrow \infty
$$

as $z \rightarrow w_{0}$ in $\Omega$.

As an application of Theorem 1, we obtain following

Corollary 1. Let $\Omega$ be a bounded domain in $\mathbf{C}$ with $\bar{\Omega}^{\circ}=\Omega$. Then the Bergman metric can not be extended beyond $\Omega$.

It is well known that the Bergman kernel function and the Bergman metric of $\Omega \backslash A$ can be extended through $A$ if $A$ is an complex analytic subvariety of dimension $\leq n-1$. For any bounded pseudoconvex $\Omega$ in $\mathbf{C}^{n}$ with $\bar{\Omega}^{\circ}=\Omega$, Pflug [23] proved that the Bergman kernel function can not be extended through any boundary point. However, this phenomenon dose not always occur for the Bergman metric in the case $n \geq 2$. For example, the Hartogs triangle $\Omega=\left\{\left(z_{1}, z_{2}\right) \in \mathbf{C}^{2}:\left|z_{1}\right|<\left|z_{2}\right|<1\right\}$ satisfies $\bar{\Omega}^{\circ}=\Omega$; nevertheless, it is well-known that the Bergman metric can be continued across the origin. It would be interesting to find sufficient conditions for the Bergman metric not being extended through the boundary.

When the psh peak function in Theorem 1 satisfies certain growth condition, one can even obtain a quantitative estimate for the Bergman metric:

Theorem 2. Let $\Omega$ be a bounded pseudoconvex domain in $\mathbf{C}^{n}$. Suppose there exist positive constants $c, \alpha, \gamma$ such that for each $p \in \partial \Omega$ there is a psh peak function $\rho_{p}$ for $\Omega$ at $p$ verifying

$$
|z-p|^{\alpha} \leq-\rho_{p}(z) \leq c|z-p|^{\gamma}
$$

for all $z \in \bar{\Omega}$. Then there exist a number $\tau>0$ and a constant $C>0$ such that

$$
B_{\Omega}(z ; X) \geq C|X| / \delta_{\Omega}^{\tau}(z),
$$

where $\delta_{\Omega}(z)$ is the euclidean boundary distance of $z$. 
Remarks. (1) The assumptions are satisfied for pseudoconvex domains with real analytic boundary [9] or more generally, pseudoconvex domains of finite type in $\mathbf{C}^{n}$ [4]. In both cases such an estimate has also been obtained by Diederich et. al. [10] and McNeal [20];

(2) We are not going to determine the exponent $\tau$ precisely.

The domains in Theorem 1 or 2 are quite general. We provide in the last section some examples of non-smooth pseudoconvex domains on which the hypothesis of Theorem 1 or 2 is satisfied. The defining equation is $\left|z_{1}\right|^{2 / \alpha_{1}}+\left|z_{2}\right|^{2 / \alpha_{2}}+\cdots+\left|z_{n}\right|^{2 / \alpha_{n}}+\varphi(z)<0$ where $\alpha_{j}>0, j=1,2, \ldots, n$ and $\varphi$ is a Hölder continuous psh function in $\mathbf{C}^{n}$.

In the proof of the Bergman completeness for hyperconvex domains, the boundary behavior of the pluricomplex Green function plays an important role. Motivated by this fact, we shall prove that (1) also holds under a hypothesis characterized by the pluricomplex Green function. We begin with some notions.

Definition. The pluricomplex Green function of $\Omega$ is defined by

$$
\begin{array}{r}
g_{\Omega}(z, w)=\sup \{\varphi(z): \varphi \text { is psh on } \Omega, \varphi \leq 0 \\
\varphi(z) \leq \log |z-w|+O(1)\} .
\end{array}
$$

This definition was firstly given by Klimek [18]. It coincides with the (negative) Green function for the Laplace operator in one dimensional case. The function $g_{\Omega}(\cdot, w)$ is a negative psh function in $\Omega$ and it has a logarithmic pole at $w$. It is also invariant under biholomorphic mappings. If $\Omega$ is hyperconvex, then $g_{\Omega}(z, w) \rightarrow 0$ as $z \rightarrow \partial \Omega$ and it is continuous on $\bar{\Omega} \times \Omega$. This result is due to Demailly [8]. The pluricomplex Green function plays a similar role in the pluripotential theory as the Green function in the classical potential theory.

Coman introduced in [5] the following

Definition. We say that $\Omega$ has property $(P)$ at $w_{0} \in \partial \Omega$ if for every compact set $K \subset \bar{\Omega} \backslash\left\{w_{0}\right\}$ one has $g_{\Omega}(z, w) \rightarrow 0$ as $w \rightarrow w_{0}$, uniformly for $z \in K \cap \Omega$.

We shall show

TheOREM 3. Let $\Omega$ be a bounded pseudoconvex domain in $\mathbf{C}^{n}$ and let $w_{0} \in \partial \Omega$. Suppose that $\Omega$ has property $(P)$ at $w_{0}$. Then $(1)$ holds. 
This class of domains contains the following cases (cf. [5]):

(1) $w_{0}$ is a local weak peak point for $\Omega$, i.e., there are a neighborhood $U$ of $w_{0}$ and a holomorphic map $h: \Omega \cap U \rightarrow \Delta$, where $\Delta$ is the unit disc in the complex plane, so that $\lim _{z \rightarrow w_{0}}|h(z)|=1$ and $\limsup _{z \rightarrow q}|h(z)|<1$, for every $q \in \partial \Omega, q \neq w_{0}$;

(2) $g_{\Omega}$ is symmetric, that is, $g_{\Omega}(z, w)=g_{\Omega}(w, z)$ for all $z, w \in \Omega$ (this case involves all bounded domains in $\mathbf{C}$, convex domains and homogeneous domains in $\mathbf{C}^{n}[8]$ ), and there exists a psh peak function $\rho$ for $\Omega$ at $w_{0}$;

(3) There is a psh peak function $\rho$ for $\Omega$ at $w_{0}$ such that

(i) $\rho$ is Hölder continuous at $w_{0}$;

(ii)

$$
\begin{aligned}
N_{\rho}(r) & :=\max \left\{\frac{\log |\rho(z)|}{\log \left|z-w_{0}\right|}: z \in \bar{\Omega}, r \leq\left|z-w_{0}\right| \leq 1 / 2\right\} \\
& =O(\log \log (1 / r))
\end{aligned}
$$

as $r \rightarrow 0$.

The proofs of the theorems are based on the $L^{2}$-estimates of the $\bar{\partial}$ operator firstly introduced by Donnelly-Fefferman [13] and then generalized by Diederich-Ohsawa [11], [12] and Berndtsson [1]. The proof of the main theorem is inspired especially by Diederich-Ohsawa [11].

\section{$\S 2$. Some $L^{2}$-estimates for the $\bar{\partial}$-operator}

In this section, we recall some powerful $L^{2}$-tools for the $\bar{\partial}$-equation. The following brilliant result is due to Donnelly-Fefferman [13]:

Proposition 4. Let $\Omega$ be a bounded pseudoconvex domain in $\mathbf{C}^{n}$ and let $\varphi$ be psh in $\Omega$. Let $\psi$ be psh and assume that $\partial \bar{\partial} \psi \geq \partial \psi \bar{\partial} \psi$ holds in the distribution sense (equivalently, the function $e^{-\psi}$ is plurisuperharmonic). Then for any $\bar{\partial}$-closed $(0,1)$-form $g$ in $\Omega$ there is a solution to the equation $\bar{\partial} u=g$ such that

$$
\int_{\Omega}|u|^{2} e^{-\varphi} d V_{n} \leq C \int_{\Omega}|g|_{\partial \bar{\partial} \psi}^{2} e^{-\varphi} d V_{n}
$$

Here $C>0$ is a numerical constant and $d V_{n}$ denotes the Lebesgue measure in $\mathbf{C}^{n}$. 
Important generalizations of this result have been done by DiederichOhsawa [11], [12] and Berndtsson [1]. We use the version of Berndtsson:

Proposition 5. Let $\Omega$ be a bounded pseudoconvex domain in $\mathbf{C}^{n}$ and let $\varphi, \psi$ be as in Porposition 4. Let $0<\nu<1$. Then for any $\bar{\partial}$-closed $(0,1)$-form $g$ in $\Omega$ there is a solution $u$ to the equation $\bar{\partial} u=g$ such that

$$
\int_{\Omega}|u|^{2} e^{-\varphi+\nu \psi} d V_{n} \leq \frac{4}{\nu(1-\nu)^{2}} \int_{\Omega}|g|_{\partial \bar{\partial} \psi}^{2} e^{-\varphi+\nu \psi} d V_{n} .
$$

\section{§3. Proof of Theorem 3}

We begin with some notions. Let $\Omega$ be a bounded pseudoconvex domain in $\mathbf{C}^{n}$ and let $\varphi$ be a psh function on $\Omega$. By $L^{2}(\Omega)$ we denote all the squareintegrable functions on $\Omega$. The $L^{2}$ norm is denoted by $\|\cdot\|_{\Omega}$. Let $H^{2}(\Omega)$ denote the subspace of holomorphic functions in $L^{2}(\Omega)$. We define for any measurable function on $\Omega$ :

$$
\|f\|_{\Omega, \varphi}^{2}:=\int_{\Omega}|f|^{2} e^{-\varphi} d V_{n}
$$

We say that $f \in L^{2}(\Omega, \varphi)$ if $\|f\|_{\Omega, \varphi}<\infty$. Now we are going to prove Theorem 3. Without loss of generality, we may assume $w_{0}=0$ and $\Omega \subset$ $\{|z|<1\}$. By the hypothesis of the theorem, for each $0<\epsilon<1$ there exists a number $0<\delta<<\epsilon$ so that

$$
g_{\Omega}(z, w) \geq-1
$$

for all $z, w \in \Omega$ with $|z| \geq \epsilon$ and $|w| \leq \delta$. Let $\chi: \mathbf{R} \rightarrow[0,1]$ be a $C^{\infty}$ function satisfying $\chi \equiv 1$ on $(-\infty, 1)$ and $\chi \equiv 0$ on $(1+\log 2, \infty)$. We put

$$
\begin{aligned}
\psi(z) & =-\log (-\log |z|) \\
\eta_{\epsilon}(z) & =\chi(\psi(z)+\log (-\log \epsilon)+1) \\
\varphi(z) & =2(n+1) g_{\Omega}(z, w)+\frac{1}{2} \psi(z) .
\end{aligned}
$$

Now fix $w \in \Omega$ with $|w| \leq \delta$ for a moment. Let $X \in T^{1,0}\left(\mathbf{C}^{n}\right)$. Since the Bergman metric is biholomorphically invariant, we may assume $X=$ $|X| \partial / \partial z_{1}$ for the sake of simplicity. Since $\psi$ is psh on $\Omega$ and satisfies $\partial \bar{\partial} \psi \geq$ $\partial \psi \bar{\partial} \psi$, there is, according to Proposition 4 , a solution to the $\bar{\partial}$-equation

$$
\bar{\partial} u_{\epsilon}=\left(z_{1}-w_{1}\right) \frac{K_{\Omega}(z, w)}{K_{\Omega}^{1 / 2}(w)} \bar{\partial} \eta_{\epsilon}(z)
$$


together with the estimate

$$
\begin{aligned}
& \int_{\Omega}\left|u_{\epsilon}\right|^{2} e^{-2(n+1) g_{\Omega}(z, w)} d V_{n} \\
\leq & C \int_{\Omega}\left|z_{1}-w_{1}\right|^{2}\left|K_{\Omega}(z, w)\right|^{2} / K_{\Omega}(w)\left|\bar{\partial} \eta_{\epsilon}\right|_{\partial \bar{\partial} \psi} e^{-2(n+1) g_{\Omega}(z, w)} d V_{n} \\
\leq & C \sup \left|\chi^{\prime}\right|^{2} \int_{\Omega \cap\left\{\epsilon \leq|z| \leq \epsilon^{1 / 2}\right\}}|z-w|^{2}\left|K_{\Omega}(z, w)\right|^{2} / K_{\Omega}(w) e^{-2(n+1) g_{\Omega}(z, w)} d V_{n} \\
\leq & C e^{2(n+1)} \sup \left|\chi^{\prime}\right|^{2} \int_{\Omega \cap\left\{|z| \leq \epsilon^{1 / 2}\right\}}|z-w|^{2}\left|K_{\Omega}(z, w)\right|^{2} / K_{\Omega}(w) d V_{n} \\
\leq & C_{1} \epsilon
\end{aligned}
$$

where $C_{1}$ only depends on $n, \sup \left|\chi^{\prime}\right|$. It follows that $u_{\epsilon}(w)=0$ and $\partial u_{\epsilon} / \partial z_{1}(w)=0$ since $g_{\Omega}(z, w) \sim \log |z-w|$ as $z \rightarrow w$. Let

$$
f_{\epsilon}(z)=\left(z_{1}-w_{1}\right) \eta_{\epsilon}(z) K_{\Omega}(z, w) / K_{\Omega}^{1 / 2}(w)-u_{\epsilon}(z)
$$

Then $f_{\epsilon}$ is holomorphic on $\Omega$. Since $|w|<\delta<\epsilon$, one has $\eta_{w}(w)=1$. Hence, $f_{\epsilon}(w)=0, X f_{\epsilon}(w)=|X| K_{\Omega}^{1 / 2}(w)$ and

$$
\begin{aligned}
\int_{\Omega}\left|f_{\epsilon}\right|^{2} d V_{n} & \leq 2 \int_{\Omega}\left|\left(z_{1}-w_{1}\right) \eta_{\epsilon}(z) K_{\Omega}(z, w) / K_{\Omega}^{1 / 2}(w)\right|^{2} d V_{n}+2 \int_{\Omega}\left|u_{\epsilon}\right|^{2} d V_{n} \\
& \leq C_{2} \epsilon+2 \int_{\Omega}\left|u_{\epsilon}\right|^{2} e^{-2(n+1) g_{\Omega}(z, w)} d V_{n} \\
& \leq C_{3} \epsilon
\end{aligned}
$$

because $g_{\Omega}(z, w)<0$ on $\Omega$. Here the positive constants only depend on $n$ and $\sup \left|\chi^{\prime}\right|$. We set $h_{\epsilon}=f_{\epsilon} /\left\|f_{\epsilon}\right\|_{\Omega}$. From the well-known formula of the Bergman metric

$B_{\Omega}(w ; X)=K_{\Omega}^{-1 / 2}(w) \sup \left\{|X f(w)|: f \in H^{2}(\Omega), f(w)=0\right.$ and $\left.\|f\|_{\Omega} \leq 1\right\}$ we obtain

$$
B_{\Omega}(w ; X) \geq \frac{\left|X h_{\epsilon}(w)\right|}{K_{\Omega}^{1 / 2}(w)}=\frac{\left|X f_{\epsilon}(w)\right|}{K_{\Omega}^{1 / 2}(w)\left\|f_{\epsilon}\right\|_{\Omega}} \geq C_{3}^{-1 / 2} \epsilon^{-1 / 2}|X|
$$

for any $w \in \Omega \cap\{|w|<\delta\}$. This proves the theorem. 


\section{§4. Proofs of Theorems 1, 2}

We assume $c=1$ for the sake of simplicity (otherwise, we replace $\rho$ by $\rho / c)$. Put $\phi=-\log (-\rho)$. Without loss of generality, we may assume $\rho(z)>-1 / 2$ on $\Omega$ and $\operatorname{diam} \Omega \leq e^{-1}$. This implies $\phi>\log 2$ on $\Omega$. Choose a $C^{\infty}$ cut-off function (we still denote $\chi$ for the sake of simplicity) so that $\left.\chi\right|_{(-\infty, 0)} \equiv 1,\left.\chi\right|_{(1, \infty)} \equiv 0, \sup \left|\chi^{\prime}\right| \leq 2$ and $\sup \left|\chi^{\prime \prime}\right| \leq 1$. Let $0<\epsilon<<1$ be an arbitrary fixed number. We define on $\Omega$ a function as follows:

$$
g_{k, w}(z)=\chi\left(\frac{1}{\log k}(-\log \phi(z)+\log (-\log \epsilon))+1\right) \log |z-w|
$$

for any $k>1$ and $w \in \Omega$. The following lemma is similar to Proposition 2.1 in Diederich-Ohsawa [11].

LEMma 6. There exists a constant $k_{0}>1$ (only depends on $n, \gamma$ ) so that for any $w \in \Omega$ with $\left|w-w_{0}\right|<\frac{1}{2} \epsilon^{k_{0} / \gamma}$ the following holds

(i) $g_{k_{0}, w} \sim \log |z-w|$ near $w$;

(ii) $g_{k_{0}, w}(z)+\frac{1}{8(n+1)}(\phi(z)-\log (-\log |z-w|))$ is a psh function on $\Omega$.

Proof. We assume $\left|w-w_{0}\right|<\frac{1}{2} \epsilon^{k / \gamma}$ where $k$ will be determined later. Then one has

$$
\rho(w) \geq-\left|w-w_{0}\right|^{\gamma}>-\frac{1}{2^{\gamma}} \epsilon^{k}
$$

Since

$$
\left\{z \in \Omega: g_{k, w}(z)=\log |z-w|\right\} \supset\left\{z \in \Omega: \rho(z)>-\epsilon^{k}\right\},
$$

one has $g_{k, w} \sim \log |z-w|$ near $w$. By a straightforward computation, we obtain the following equality in the distribution sense

$$
\begin{aligned}
& \partial \bar{\partial} g_{k, w}=\frac{\log |z-w|}{\phi \log k}\left(\chi^{\prime \prime}(\cdot) \frac{\partial \phi \bar{\partial} \phi}{\phi \log k}+\chi^{\prime}(\cdot) \frac{\partial \phi \bar{\partial} \phi}{\phi}-\chi^{\prime}(\cdot) \partial \bar{\partial} \phi\right) \\
& -\frac{\chi^{\prime}(\cdot) \log |z-w|}{\phi \log k}\left(\partial \phi \frac{\bar{\partial} \log |z-w|}{\log |z-w|}+\bar{\partial} \phi \frac{\partial \log |z-w|}{\log |z-w|}\right) \\
& +\chi(\cdot) \partial \bar{\partial} \log |z-w| \text {. }
\end{aligned}
$$

Observe that

$$
\begin{aligned}
\operatorname{supp} \chi^{\prime}(\cdot) & \subset\left\{z \in \Omega: \rho(z) \leq-\epsilon^{k}\right\} \\
& \subset\left\{z \in \Omega:\left|z-w_{0}\right| \geq \epsilon^{k / \gamma}\right\} .
\end{aligned}
$$


It follows that

$$
|z-w| \geq\left|z-w_{0}\right|-\left|w-w_{0}\right| \geq \frac{1}{2}\left|z-w_{0}\right|
$$

on $\operatorname{supp} \chi^{\prime}(\cdot)$ since $\left|w-w_{0}\right|<\frac{1}{2} \epsilon^{k / \gamma}$. Hence

$$
\begin{aligned}
|\phi(z)| & =|\log (-\rho(z))| \\
& \geq \gamma|\log | z-w_{0}|| \\
& \geq \gamma|\log (2|z-w|)|
\end{aligned}
$$

holds there. By Cauchy-Schwarz's inequality, the following inequality holds in the distribution sense

$$
\pm 2 \operatorname{Re} \frac{\partial \phi \bar{\partial} \log |z-w|}{\log |z-w|} \geq-\partial \phi \bar{\partial} \phi-\partial \log (-\log |z-w|) \bar{\partial} \log (-\log |z-w|) .
$$

Then we can find, by neglecting the semipositive term $\chi(\cdot) \partial \bar{\partial} \log |z-w|$, a constant $C^{\prime}>0$ (only depends on $\gamma$ ) so that

$$
\partial \bar{\partial} g_{k, w}(z) \geq-\frac{C^{\prime}}{\log k}(\partial \bar{\partial} \phi+\partial \bar{\partial}(-\log (-\log |z-w|)))
$$

provided $k \geq e$, because $\phi>\log 2$ on $\Omega, \partial \bar{\partial} \phi \geq \partial \phi \bar{\partial} \phi$ and

$$
\partial \bar{\partial}(-\log (-\log |z-w|)) \geq \partial \log (-\log |z-w|) \bar{\partial} \log (-\log |z-w|) .
$$

To complete the proof we only need to take $k_{0}$ so large that $C^{\prime} / \log k_{0} \leq$ $\frac{1}{8(n+1)}$.

Proof of Theorem 1. Let $0<\epsilon<<1$ and $w \in \Omega$ with $g_{k_{0}, w}$ be as above. We denote by $g_{w}=g_{k_{0}, w}$ for the sake of simplicity. Let

$$
\delta=\delta(\epsilon):=\sup _{z \in \bar{\Omega}, \rho(z) \geq-\epsilon}\left|z-w_{0}\right| .
$$

Then $\delta>0$ and $\delta \rightarrow 0$ as $\epsilon \rightarrow 0$ since $\rho$ is a psh peak function. Let $\kappa: \mathbf{R} \rightarrow[0,1]$ such that $\left.\kappa\right|_{(-\infty, 1-\log 2)}=1$ and $\left.\kappa\right|_{(1, \infty)}=0$. We put

$$
\begin{aligned}
\eta_{w} & =\kappa\left(-\log \left(-\log \left|z-w_{0}\right|\right)+\log \left(-\log \delta^{1 / 2}\right)+1\right) \\
\psi_{w} & =\frac{1}{2}\left(\phi-\log \left(-\log \left|z-w_{0}\right|\right)\right) \\
\varphi_{w} & =2(n+1) g_{w}-\frac{1}{4} \log (-\log |z-w|)+\frac{1}{2} \psi_{w} .
\end{aligned}
$$


By Lemma $6, \varphi_{w}$ is psh on $\Omega$. Obviously one has $\partial \bar{\partial} \psi_{w} \geq \partial \psi_{w} \bar{\partial} \psi_{w}$ and $\partial \bar{\partial} \psi_{w} \geq \frac{1}{2} \partial \log \left(-\log \left|z-w_{0}\right|\right) \bar{\partial} \log \left(-\log \left|z-w_{0}\right|\right)$. This implies

$$
\left|\bar{\partial} \eta_{w}\right|_{\partial \bar{\partial} \psi_{w}} \leq \sqrt{2} \sup \left|\kappa^{\prime}\right|
$$

Notice that

$$
\begin{aligned}
& \operatorname{supp} g_{w} \subset\{z \in \Omega: \rho(z) \geq-\epsilon\} \\
& \subset\left\{z \in \Omega: \eta_{w}(z)=1\right\} .
\end{aligned}
$$

In particular, one has $\eta_{w}(w)=1$. Let $X=|X| \partial / \partial z_{1}$. We apply Proposition 5 with $\nu=1 / 2, \varphi=\varphi_{w}, \psi=\psi_{w}$ to solve the $\bar{\partial}$-equation

$$
\bar{\partial} u_{w}=\left(z_{1}-w_{1}\right) K_{\Omega}(z, w) / K_{\Omega}^{1 / 2}(w) \bar{\partial} \eta_{w}
$$

on $\Omega$ with the estimate

$$
\begin{aligned}
& \int_{\Omega}\left|u_{w}\right|^{2} e^{-2(n+1) g_{w}+\frac{1}{4} \log (-\log |z-w|)} d V_{n} \\
\leq & 32 \int_{\Omega_{t}}\left|z_{1}-w_{1}\right|^{2}\left|K_{\Omega}(z, w) / K_{\Omega}^{1 / 2}(w)\right|^{2}\left|\bar{\partial} \eta_{w}\right|_{\partial \bar{\partial} \psi_{w}}^{2} \\
& e^{-2(n+1) g_{w}+\frac{1}{4} \log (-\log |z-w|)} d V_{n} \\
\leq & C_{1} \int_{\Omega \cap\left\{\left|z-w_{0}\right|<\delta^{1 / 2}\right\}}|z-w|^{2}(-\log |z-w|)^{1 / 4} \\
& \left|K_{\Omega}(z, w) / K_{\Omega}^{1 / 2}(w)\right|^{2} d V_{n} \\
\leq & C_{2} \delta^{1 / 2}
\end{aligned}
$$

because supp $g_{w} \cap \operatorname{supp} \bar{\partial} \eta_{w}=\emptyset$. Here $C_{1}, C_{2}>0$ are constants only depend on $\sup \left|\kappa^{\prime}\right|$. Since

$$
2(n+1) g_{w}-\frac{1}{4} \log (-\log |z-w|)<0
$$

on $\Omega$ and

$$
2(n+1) g_{w}-\frac{1}{4} \log (-\log |z-w|)<2(n+1) \log |z-w|
$$

near $w$, the function $f_{w}=\left(z_{1}-w_{1}\right) \eta_{w} K_{\Omega}(z, w) / K_{\Omega}^{1 / 2}(w)-u_{w}$ is holomorphic on $\Omega$ and satisfies

$$
f_{w}(w)=u_{w}(w)=0, X f_{w}(w)=|X| K_{\Omega}^{1 / 2}(w)
$$


and

$$
\begin{aligned}
\left\|f_{w}\right\|_{\Omega} & \leq\left\|\left(z_{1}-w_{1}\right) \eta_{w} K_{\Omega}(\cdot, w) / K_{\Omega}^{1 / 2}(w)\right\|_{\Omega}+\left\|u_{w}\right\|_{\Omega} \\
& \leq C_{3} \delta^{1 / 2}+\left\|u_{w}\right\|_{\Omega, 2(n+1) g_{w}-\frac{1}{4} \log (-\log |z-w|)} \\
& \leq C_{4} \delta^{1 / 4} .
\end{aligned}
$$

This implies

$$
B_{\Omega}(w ; X) \geq C_{4}^{-1} \delta^{-1 / 4}|X|
$$

for any $w \in \Omega$ with $\left|z-w_{0}\right|<\frac{1}{2} \epsilon^{k_{0} / \gamma}$. This proves the theorem.

Proof of Theorem 2. It suffices to modify the proof of Theorem 1 slightly. Let $w \in \Omega$ be fixed for a moment. Choose a boundary point $w^{\prime}$ so that $\left|w-w^{\prime}\right|=\delta_{\Omega}(w)$. We take $\epsilon=\left(3 \delta_{\Omega}(w)\right)^{\gamma / k_{0}}$. We replace the $w_{0}$ by $w^{\prime}, \rho$ by $\rho_{w^{\prime}}$ and repeat the argument as in the proof of Theorem 1. Clearly one has $\left|w-w^{\prime}\right|<\frac{1}{2} \epsilon^{k_{0} / \gamma}$ and

$$
\delta=\sup _{z \in \bar{\Omega}, \rho_{w^{\prime}}(z) \geq-\epsilon}\left|z-w^{\prime}\right| \leq c^{\prime} \delta_{\Omega}^{\frac{\gamma}{\alpha k_{0}}}(w)
$$

because $-\rho_{w^{\prime}}(z) \geq\left|z-w^{\prime}\right|^{\alpha}$. We obtain finally the following

$$
B_{\Omega}(w ; X) \geq C \delta_{\Omega}^{-\frac{\gamma}{4 \alpha k_{0}}}(w)|X|
$$

for suitable constant $C>0$. To complete the proof, we only need to take $\tau=\frac{\gamma}{4 \alpha k_{0}}$.

Proof of the corollary. Let $w_{0} \in \partial \Omega$ be an arbitrary point. Then there exist a sequence of points $w_{k} \in \mathbf{C} \backslash \bar{\Omega}, k=1,2, \ldots$ which converge to $w_{0}$ as $k \rightarrow \infty$. Let $w_{k}^{\prime} \in \partial \Omega$ be the nearest point to $w_{k}$ for each $k$. It follows that $\Omega \subset \mathbf{C} \backslash \Delta\left(w_{k}, r_{k}\right)$ where $r_{k}=\left|w_{k}-w_{k}^{\prime}\right|$ and $\Delta(x, r)$ denotes the disc which is centered at $x$ with radius $r$. Since $\partial \Delta\left(w_{k}, r_{k}\right)$ is $C^{\infty}, \Omega$ admits a Hölder continuous psh peak function at $w_{k}^{\prime}$ for each $k$. According to Theorem 1 , the Bergman metric goes to infinity uniformly at $w_{k}$. This implies that the Bergman metric can not be continuously extended across $w_{0}$.

\section{§5. Examples and remarks}

1. Let $\varphi$ be a Hölder continuous psh function in $\mathbf{C}^{n}$. We consider the following domain

$$
\Omega=\left\{z \in \mathbf{C}^{n}: r(z)=\left|z_{1}\right|^{2 / \alpha_{1}}+\left|z_{2}\right|^{2 / \alpha_{2}}+\cdots+\left|z_{n}\right|^{2 / \alpha_{n}}+\varphi(z)<0\right\}
$$


where $\alpha_{j}>0, j=1,2, \ldots, n$. If $\varphi \equiv-1$, then $\Omega$ is a well-known Reinhardt domain which has been studied by many authors (cf. [6], [7], [14]). Now let $p$ be any boundary point. Without loss of generality, we may assume $p_{j} \neq 0$ if $1 \leq j \leq l$ and $p_{j}=0$ if $j>l$ for some positive integer $l$. We take an open neighborhood $U$ of $p$ so that $z_{j}$ does not vanish there for $1 \leq j \leq l$. For each $z \in \Omega \cap U$ one has

$$
\begin{aligned}
-r(z)+ & \left|z_{j}\right|^{2 / \alpha_{j}}>\left|z_{j}^{1 / \alpha_{j}}\right|^{2} \\
& =\left|z_{j}^{1 / \alpha_{j}}-p_{j}^{1 / \alpha_{j}}\right|^{2}+2 \operatorname{Re} \bar{p}_{j}^{1 / \alpha_{j}}\left(z_{j}^{1 / \alpha_{j}}-p_{j}^{1 / \alpha_{j}}\right)+\left|p_{j}\right|^{2 / \alpha_{j}}
\end{aligned}
$$

for $1 \leq j \leq l$. Put

$$
\rho_{j}(z)= \begin{cases}r(z)-\left|z_{j}\right|^{2 / \alpha_{j}}+2 \operatorname{Re} \bar{p}_{j}^{1 / \alpha_{j}}\left(z_{j}^{1 / \alpha_{j}}-p_{j}^{1 / \alpha_{j}}\right)+\left|p_{j}\right|^{2 / \alpha_{j}} & \text { if } 1 \leq j \leq l \\ r(z)-\left|z_{j}\right|^{2 / \alpha_{j}} & \text { if } j>l\end{cases}
$$

Notice that

$r(z)-\left|z_{j}\right|^{2 / \alpha_{j}}=\left|z_{1}\right|^{2 / \alpha_{1}}+\cdots+\left|z_{j-1}\right|^{2 / \alpha_{j-1}}+\left|z_{j+1}\right|^{2 / \alpha_{j+1}}+\cdots+\left|z_{n}\right|^{2 / \alpha_{n}}+\varphi(z)$.

It follows that $\rho_{j}$ is psh on $\Omega \cap U$ for each $j$ and satisfies

$$
\rho_{j}(z) \leq \begin{cases}-\left|z_{j}^{1 / \alpha_{j}}-p_{j}^{1 / \alpha_{j}}\right|^{2} & \text { if } 1 \leq j \leq l \\ -\left|z_{j}\right|^{2 / \alpha_{j}} & \text { if } j>l\end{cases}
$$

Let $\rho=\sum_{j=1}^{n} \rho_{j}$. Then $\rho$ is a psh peak function for $\Omega \cap U$ at $p$ and clearly, it is Hölder continuous in $U$. To get the global psh peak function, we take $\tilde{\rho}=\max \{\rho,-\delta\}$ for suitable fixed constant $\delta>0$, and extend $\rho$ as constant $-\delta$ where it is not defined. Thus Theorem 1 applies.

2 . Let $\Omega$ be defined as above. Furthermore, we assume $0<\alpha_{j} \leq 1$ for all $1 \leq j \leq n$. Let $p$ be any boundary point. Note that for any $z \in \Omega$ one has

$$
\left(-r(z)+\left|z_{j}\right|^{2 / \alpha_{j}}\right)^{\alpha_{j}}>\left|z_{j}\right|^{2}=\left|z_{j}-p_{j}\right|^{2}+2 \operatorname{Re} \bar{p}_{j}\left(z_{j}-p_{j}\right)+\left|p_{j}\right|^{2}
$$

if $1 \leq j \leq l$. Set

$$
\rho_{j, p}=\left|p_{j}\right|^{2}+2 \operatorname{Re} \bar{p}_{j}\left(z_{j}-p_{j}\right)-\left(-r(z)+\left|z_{j}\right|^{2 / p_{j}}\right)^{\alpha_{j}}
$$

Clearly $\rho_{j, p}$ is psh since $0<\alpha_{j} \leq 1$, and also $-\rho_{j, p}(z)>\left|z_{j}-p_{j}\right|^{2}$ holds on $\Omega$. It follows that the function $\rho_{p}:=\sum_{j=1}^{n} \rho_{j, p}$ is a psh peak function which satisfies the hypothesis of Theorem 2 because $r$ is Hölder continuous in $\mathbf{C}^{n}$. Then Theorem 2 applies. 
Remarks. If we let $\varphi \equiv-1$ in the above example, then $\Omega$ is convex and the function defined by

$$
\tilde{r}(z)=\left(\sup _{p \in \partial \Omega}\left(\rho_{p}(z)+|z-p|^{2}\right)\right)^{*}
$$

(where $*$ denotes the uppersemicontinuous regularization) is a psh function on $\Omega$ with the following holds

(i) $-C \delta_{\Omega}^{\alpha}(z) \leq \tilde{r}(z)<0$ where $\alpha=\min \left\{\alpha_{1}, \alpha_{2}, \ldots, \alpha_{n}\right\}$;

(ii) $\partial \bar{\partial} \tilde{r} \geq \partial \bar{\partial}|z|^{2}$ holds in the distribution sense.

Due to a result of Sibony [24], we may bound the Kobayashi metric from below by $C|X| / \delta_{\Omega}^{\alpha / 2}(z)$. Combining the well-known fact that the Caratheodory metric coincides with the Kobayashi metric for convex domains [8] together with the fact that the Bergman metric is always no less than the Caratheodory metric [15], we immediately obtain a sharp estimate for the Bergman metric

$$
B_{\Omega}(z ; X) \geq C|X| / \delta_{\Omega}^{\alpha / 2}(z)
$$

3. In [11], Diederich-Ohsawa obtained a quantitative estimate for the Bergman distance for bounded pseudoconvex domains in $\mathbf{C}^{n}$, on which there exists a bounded $C^{\infty}$ strictly psh exhaustion function $\rho$ on $\Omega$ such that

$$
\frac{1}{c_{1}} \delta_{\Omega}^{1 / c_{2}}(z) \leq-\rho(z) \leq c_{1} \delta_{\Omega}^{c_{2}}(z)
$$

holds for suitable constants $c_{1}, c_{2}>0$. With the method we used in proving Theorem 1, the above condition can be weakened to only assume the existence of a bounded psh exhaustion function which is Hölder continuous on $\bar{\Omega}$.

Acknowledgements. The author would like to thank the referee for the suggestion which simplified the proof of Theorem 1 in the earlier version of this paper. 


\section{REFERENCES}

[1] B. Berndtsson, The extension theorem of Ohsawa-Takegoshi and the theorem of Donnelly-Fefferman, Ann. Inst. Fourier (Grenoble) , 46 (1996), 1083-1094.

[2] Z. Blocki and P. Pflug, Hyperconvexity and Bergman completeness, Nagoya Math. J. 151(1998), 221-225.

[3] B. Y. Chen, Completeness of the Bergman metric on non-smooth pseudoconvex domains, Ann. Pol. Math. LXXI (1999), 241-251.

[4] S. Cho, A lower bound on the Kobayashi metric near a point of finite type in $\mathbf{C}^{n}, \mathrm{~J}$. Geom. Anal. 2 (1992), 317-325.

[5] D. Coman, Boundary behavior of the pluricomplex Green function, Ark. Math. 36 (1998), 341-353.

[6] J. D'Angelo, A note on the Bergman kernel, Duke Math. 45 (1978), 259-265.

[7] _ An explicit computation of the Bergman kernel function, J. of Geom. Anal. 4 (1994), 23-34.

[8] J. P. Demailly, Mesures de Monge-Ampère et mesures pluriharmoniques, Math. Z. 194 (1987), 519-564.

[9] K. Diederich and J. E. Fornaess, Proper holomorphic maps onto pseudoconvex domains with real-analytic boundary, Ann. of Math. 110 (1979), 575-592.

[10] K. Diederich, J. E. Fornaess and G. Herbort, Boundary behavior of the Bergman metric, Proc. Symp. Pure Math. 41 (1984), 59-67.

[11] K. Diederich and T. Ohsawa, An estimate for the Bergman distance on pseudoconvex domains, Ann. of Math. 141 (1995), 181-190.

[12] General continuity principles for the Bergman kernel, Internat. J. of Math. 5 (1994), 189-199.

[13] H. Donnelly and C. Fefferman, $L^{2}$ - cohomology and index theorem for the Bergman metric, Ann. of Math. 118 (1983), 593-618.

[14] S. Gong and X. Zheng, The Bergman kernel of some Reinhardt domains, Trans. Amer. Math. Soc. 348 (1996), 1771-1803.

[15] K. T. Hahn, Inequality between the Bergman metric and Caratheodory differential metric, Proc. Amer. Math. Soc. 68 (1978), 193-194.

[16] G. Herbort, The Bergman metric on hyperconvex domains, Math. Z. 232 (1999), 183-196.

[17] M. Jarnicki and P. Pflug, Bergman completeness of complete circular domains, Ann. Pol. Math. 50 (1989), 219-222.

[18] M. Klimek, Extremal plurisubharmonic functions and invariant pseudodistances, Bull. Soc. Math. France. 113 (1985), 123-142.

[19] S. Kobayashi, Geometry of bounded domains, Trans. Amer. Math. Soc. 92 (1959), $267-290$.

[20] J. McNeal, Lower bounds on the Bergman metric near a point of finite type, Ann. of Math. 136 (1992), 339-360.

[21] T. Ohsawa, A remark on the completeness of the Bergman metric, Proc. Jap. Acad. Sci. 57 (1981), 283-240.

$[22] \longrightarrow$ An essay on the Bergman metric and balanced domains, Reproducing kernels 
and their applications, ISAAC, e, S. Saitoh et el. Kluwer 1999, 141-148.

[23] P. Pflug, Quadratintegrable holomorphe Functionen und die Serre-Vermutung, Math. Ann. 216 (1975), 285-288.

[24] N. Sibony, A class of hyperbolic manifolds, In: Recent Developments in Several Complex Variables, Princeton Univ. Press (1981), 357-372.

Department of Applied Mathematics

Tongli University

200092

P. R. China

chenboy@online.sh.cn 